\title{
The Tevatron Bunch by Bunch Longitudinal Dampers
}

\author{
Cheng-Yang Tan \\ Jim Steimel \\ Beams Division/Tevatron
}

\begin{abstract}
We describe in this paper the Tevatron bunch by bunch dampers. The goal of the dampers is to stop the spontaneous longitudinal beam size blowup of the protons during a store. We will go through the theory and also show the measured results during the commissioning of this system. The system is currently operational and have stopped the beam blowups during a store.
\end{abstract}




\section{INTRODUCTION}

As Run II begins its first year, unforeseen problems have started appearing which need to be fixed before higher luminosities can be achieved. One of the problems which started to appear at the beginning of 2002 is the rapid blowup of the longitudinal beam size during a store. See Figure 1. Although these blowups do not appear in every store, they seem to be weakly correlated with beam current. There are conjectures by the authors that the blowups are due to coupled bunch mode instabilities which arise from coupling to the higher order parasitic modes of the RF cavities. As these higher modes move as a function of temperature, the coupled bunch modes can be stable or unstable depending on where and how the higher order parasitic modes line up. Table 1 shows eleven stores in the month of May where about $2 / 3$ of the stores are unstable.

The first attempts at controlling this blowup with Mode 0 dampers ended in failure. This showed us that the instability must came from higher order coupled bunch modes and if we can control them, the blowups will be stopped.

After much discussion with D. McGinnis, it was decided that the best course of action is to build a bunch by bunch longitudinal damper system. At first glance, the idea of using the RF cavity themselves as the source of longitudinal kicks on the beam seems to be difficult. This is because each of the four proton RF cavities has a high $\mathrm{Q}\left(\sim 10^{4}\right)$ near its resonance and thus its impedance falls off rapidly away from it. Therefore, the amplitude and phase response is not flat at all synchrotron sideband pairs and thus the dampers are not bunch by bunch. The solution to this problem is to build an equalizer that lifts up the impedance so that it looks constant away from the resonance. Besides the equalizer, the damper also needs a notch filter which suppresses the revolution harmonics (otherwise these harmonics will limit the gain of the loop) and differentiates in time the synchrotron sidebands. Lastly, we also have to time in the system so that the error signal of bunch $n$ 


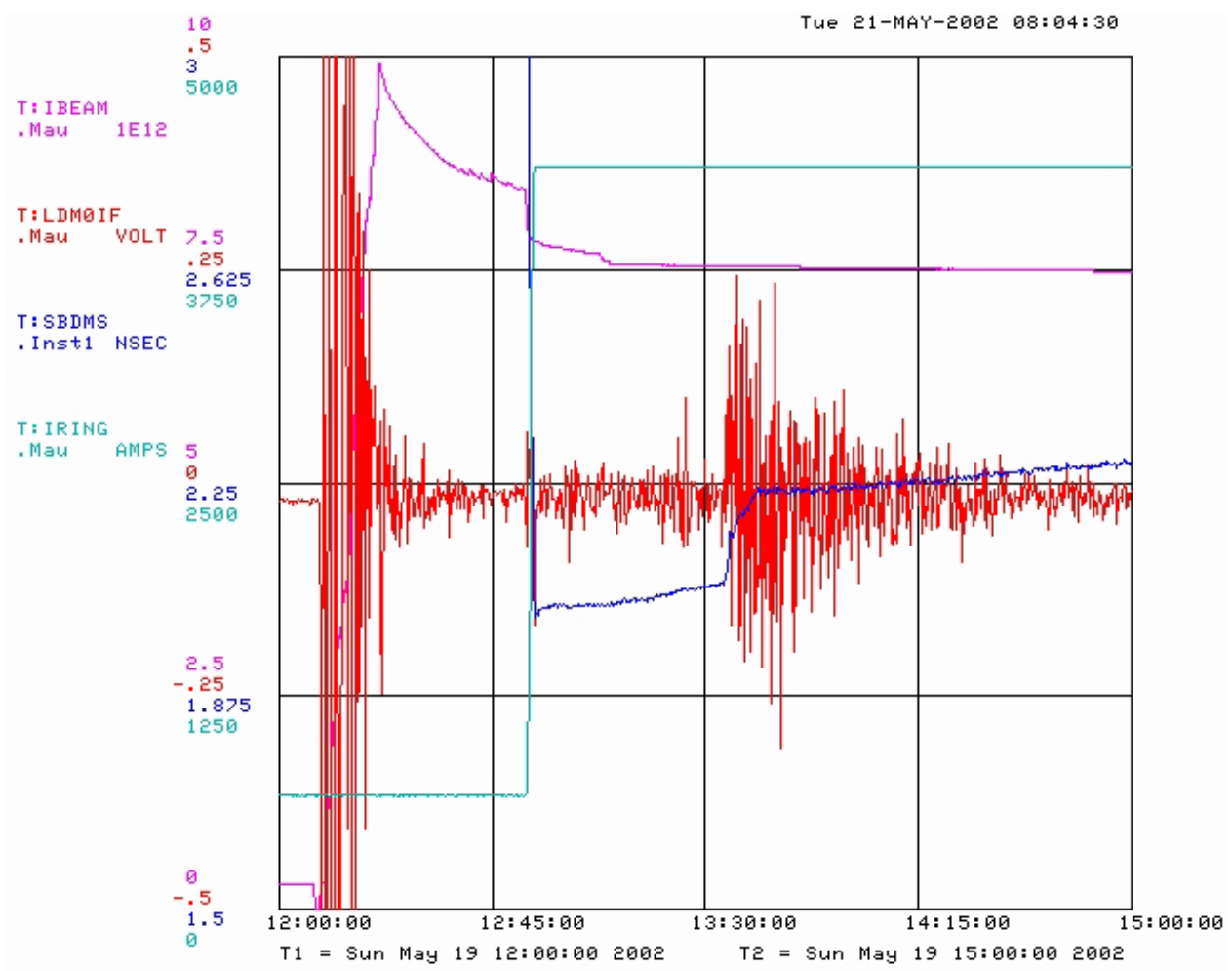

Figure 1 The beam blows up longitudinally (T:SBDMS) at about $1340 \mathrm{hrs}$ during the store which started at about $1300 \mathrm{hrs}$. We see that when it blows up the phase signal of the bunch oscillates w.r.t. RF (T:LDM0IF). Plotted also are beam current T:IBEAM and the bus current T:IRING.

\begin{tabular}{|c|c|c|c|c|c|c|c|c|}
\hline Store & date & No. protons & $\begin{array}{l}\text { bunch } \\
\text { length } \\
\text { before } \\
\text { blowup (ns) }\end{array}$ & $\begin{array}{l}\text { bunch } \\
\text { length after } \\
\text { blowup (ns) }\end{array}$ & $\begin{array}{l}\text { time before } \\
\text { blowup } \\
\text { (min) }\end{array}$ & $\begin{array}{l}\text { 1/e time } \\
\text { before } \\
\text { blowup (hr) }\end{array}$ & $\begin{array}{l}\text { 1/e time } \\
\text { after } \\
\text { blowup (hr) }\end{array}$ & comments \\
\hline 1302 & 8 May 02 & $1.70 \mathrm{E}+011$ & 2.0 & 2.3 & 60 & 42 & 67 & \\
\hline 1305 & 9 May 02 & $1.67 \mathrm{E}+011$ & 2.0 & 2.3 & 6 & 12 & 43 & \\
\hline 1307 & 10 May 02 & $1.79 \mathrm{E}+011$ & 2.0 & & & 53 & & no blow up \\
\hline 1309 & 11 May 02 & $1.71 \mathrm{E}+011$ & 2.0 & & & 42 & & no blow up \\
\hline 1313 & 12 May 02 & $1.76 \mathrm{E}+011$ & 2.0 & & & 40 & & no blow up \\
\hline 1329 & 16 May 02 & $1.76 \mathrm{E}+011$ & 1.9 & 2.2 & 3 & no data & 77 & \\
\hline 1332 & 17 May 02 & $1.78 \mathrm{E}+011$ & 1.9 & 2.4 & 6 & 9 & 83 & \\
\hline 1333 & 18 May 02 & $1.81 \mathrm{E}+011$ & 2.1 & & & 50 & & no blow up \\
\hline 1335 & 19 May 02 & $1.77 \mathrm{E}+011$ & 2.0 & 2.2 & 39 & 40 & 59 & \\
\hline 1337 & 20 May 02 & $1.83 \mathrm{E}+011$ & 2.0 & 2.2 & 16 & 19 & 56 & \\
\hline 1340 & 21 May 02 & $1.94 \mathrm{E}+011$ & 2.0 & 2.6 & 2 & no data & no data & \\
\hline
\end{tabular}

is applied exactly one turn later to kick bunch $n$.

Finally, we will like to point out that at the start of the project, we did not have any 
guarantees that our design will, in fact, work. However, we will show in this paper that this project is successful.

\begin{tabular}{c|c|c}
\hline Table 2. Parameters of the Tevatron & \multicolumn{1}{c}{ Value } \\
\hline $\begin{array}{c}\text { Symbol } \\
h\end{array}$ & $\begin{array}{c}\text { Description } \\
\text { harmonic number } \\
\text { \# of bunches }\end{array}$ & 36 \\
- & \# buckets between bunches in a train & 21 \\
- & \# buckets between trains & 140 \\
- & bucket size & $18.8 \mathrm{~ns}$ \\
\hline \hline$f_{0}$ & revolution frequency at $150 \mathrm{GeV}$ & $47.712 \mathrm{kHz}$ \\
$f_{\mathrm{RF}}$ & frequency of RF drive at $150 \mathrm{GeV}$ & $53.103639 \mathrm{MHz}$ \\
$f_{s}$ & synchrotron frequency at $150 \mathrm{GeV}$ & $88 \mathrm{~Hz}$ \\
\hline \hline$f_{0}$ & revolution frequency at $980 \mathrm{GeV}$ & $47.713 \mathrm{kHz}$ \\
$f_{\mathrm{RF}}$ & frequency of RF drive at $980 \mathrm{GeV}$ & $53.104705 \mathrm{MHz}$ \\
$f_{s}$ & synchrotron frequency at $980 \mathrm{GeV}$ & $34 \mathrm{~Hz}$ \\
\hline \hline
\end{tabular}

Before we plough on with the theory, setup and results, the relevant parameters of the Tevatron are shown in Table 2 and the Fourier transform pairs which we will use throughout this paper are

$$
\left.\begin{array}{rl}
\tilde{F}(\omega) & =\int_{-\infty}^{\infty} d t e^{-i \omega t} f(t) \\
f(t) & =\frac{1}{2 \pi} \int_{-\infty}^{\infty} d \omega e^{i \omega t} \tilde{F}(\omega)
\end{array}\right\}
$$

The choice of these Fourier transform pairs are dictated by the Hewlett Packard vector signal analyzer which we use to measure the frequency responses of the damper system. 


\section{THEORY}

Let us consider a simple damper system shown in Figure 2. The source of this derivation comes from McGinnis. Looking at Figure $2, Z_{E}$ represents the impedance of the electronics and $G_{B}$ represents the conductance of the beam. Therefore,

$$
I_{G}=G_{B} V_{\text {out }}
$$

and the output voltage $V_{\text {out }}$ of the damper is

$$
\left.\begin{array}{rl}
V_{\text {out }} & =Z_{E}\left(I_{\text {in }}+I_{G}\right) \\
& =Z_{E}\left(I_{\text {in }}+G_{B} V_{\text {out }}\right)
\end{array}\right\}
$$

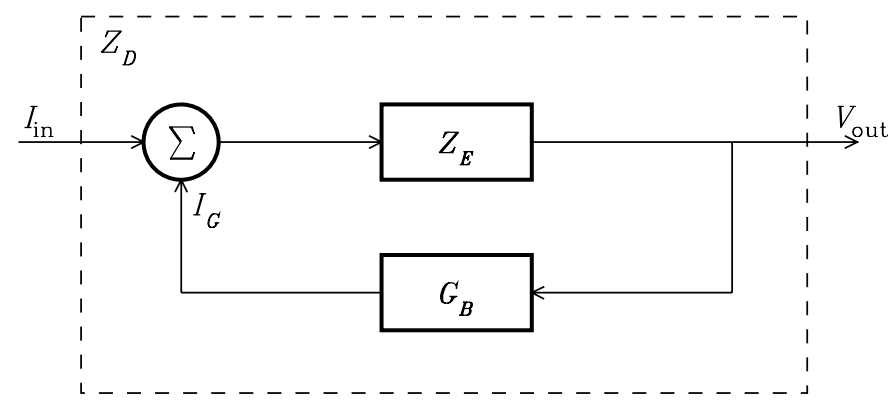

Figure 2 This is a block diagram of a simple damper system.

Solving for the impedance of the entire system $Z_{D}$, we have

$$
Z_{D}(s)=\frac{V_{\text {out }}}{I_{\text {in }}}=\frac{Z_{E}(s)}{1-G_{B}(s) Z_{E}(s)}
$$

So, if we examine (4), we can see in its denominator is $G_{B} Z_{E}$, which is the open loop response of the damper system. To determine the stability of the damper system, let $Z_{E}$ be of finite bandwidth with one pole, i.e.

$$
Z_{E} \equiv \frac{Z_{E}^{\prime}}{1+\alpha s}
$$


Then

$$
Z_{D}=\frac{Z_{E}^{\prime}}{\alpha\left(s+\frac{1-G_{B} Z_{E}^{\prime}}{\alpha}\right)}
$$

which implies that the pole is at

$$
s_{p}=-\frac{1-G_{B} Z_{E}^{\prime}}{\alpha}
$$

and thus by inverse Laplace transforming (6), we have the temporal response $W_{D}$ of the damper system

$$
\left.\begin{array}{rl}
W_{D}(t) & \sim e^{s_{p} t} \\
& =e^{-\frac{1-\operatorname{Re}\left[G_{B} Z_{E}^{\prime}\right]}{\alpha} t} \times e^{i \operatorname{Im}\left[G_{B} Z_{E}^{\prime}\right] t} \\
& =\text { (decay or growth part }) \times(\text { oscillatory part })
\end{array}\right\}
$$

Clearly, for dampers we want the decay part of (8), thus

$$
1-\operatorname{Re}\left[G_{B} Z_{E}^{\prime}\right]>0
$$

or

$$
\operatorname{Re}\left[G_{B} Z_{E}^{\prime}\right]<1
$$

which means that the real part of the open loop response must be $<1$ for damping. This is the most important result of this section. 


\section{SETUP}

In this section, we will go through each part of our setup used for our bunch by bunch

longitudinal dampers and show that the open loop response $G_{B} Z_{E}^{\prime}<1$. Figure 3 is a block diagram of the setup. The damper system starts at the stripline pickups which sum the beam signals at the two plates to produce a signal which is proportional to the longitudinal position of the beam. This signal is then mixed down with the Tevatron RF to produce a phase error (or quadrature) signal w.r.t. it. The error signal is then processed with electronics which perform the following:

(i) Equalize the impedance of the RF cavity.

(ii) Suppress the revolution harmonics and differentiate the synchrotron sidebands around the revolution lines.

(iii) One turn delay so that when the dampers pick up the signal of bunch 1 it will kick bunch 1 one turn later.

To accomplish $(i)$, we have a high pass filter (hpf) which equalizes the RF cavity impedance and for $(i i)$, we have notch filters at every revolution harmonic. For (iii) we have a digital delay and a near uniform triggering system.

\section{Equalizer}

The idea of using a hpf to equalize the impedance of the RF cavity comes from observing that if we model the RF cavity impedance $Z_{\mathrm{RF}}$ using an $R L C$ circuit (See Appendix $I V$ ) and define $R_{s}$ is its shunt impedance, $L$ is its inductance and $C$ is its capacitance, then

$$
Z_{\mathrm{RF}}=\frac{R_{s}}{1-i Q\left(\frac{\omega_{R}}{\omega}-\frac{\omega}{\omega_{R}}\right)}
$$




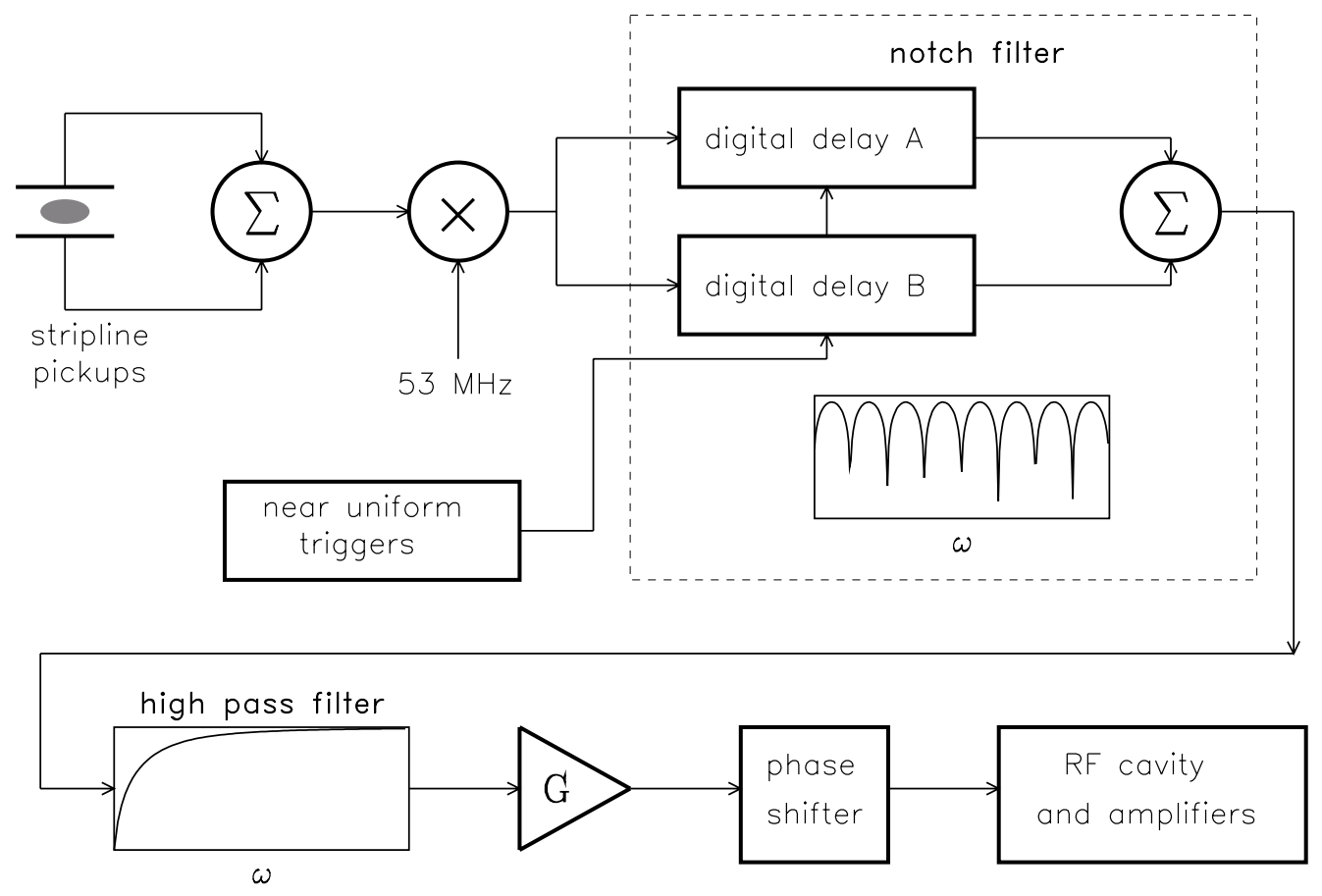

Figure 3 This figure shows the block diagram of the setup used for the longitudinal dampers.

If $\omega_{R}=1 / \sqrt{L C}$ is its resonant frequency and $Q=R_{s} \sqrt{C / L}$ is its quality factor, then the magnitude $\left|Z_{\mathrm{RF}}\right|$ is

$$
\begin{aligned}
\left|Z_{\mathrm{RF}}\right| & =\frac{R_{s}}{\sqrt{1+Q^{2}\left(\frac{\omega_{R}}{\omega}-\frac{\omega}{\omega_{R}}\right)^{2}}} \\
& =\frac{R_{s}}{Q\left|\frac{\omega_{R}}{\omega}-\frac{\omega}{\omega_{R}}\right|} \quad \text { when } Q \rightarrow \infty
\end{aligned}
$$

If we write $\omega=\omega_{R}+\delta \omega$ so that $\delta \omega / \omega_{R} \ll 1$ (for example, in our system $47 \mathrm{kHz}<\delta \omega / 2 \pi<$ $1.25 \mathrm{MHz}, \omega_{R} / 2 \pi=53 \mathrm{MHz}$, and $\left.Q \sim 10^{5}\right)$, (12) becomes

$$
\left|Z_{\mathrm{RF}}\right|=\frac{R_{s}}{2 Q}\left|\frac{\omega_{R}}{\delta \omega}\right| \sim\left|\frac{\omega_{R}}{\delta \omega}\right|
$$

which means that $\left|Z_{\mathrm{RF}}\right|$ has a $1 / \delta \omega$ type dependence when $Q \rightarrow \infty$ and $\delta \omega / \omega_{R} \ll 1$. 
Next, let us examine the response of a hpf. We introduce first a new variable $\Delta \omega=$ $\left(\omega-\omega_{\mathrm{RF}}\right)$ (the reason for doing this will become apparent later on in the analysis). $\omega_{\mathrm{RF}}$ is the $\mathrm{RF}$ drive frequency and $\omega_{\mathrm{RF}} \approx \omega_{R}$. For a hpf with a $3 \mathrm{~dB}$ response at $\Delta \omega_{3 \mathrm{~dB}}$, its response function $R_{\mathrm{hpf}}$ is

$$
R_{\mathrm{hpf}}(\Delta \omega)=\frac{1+i \frac{\Delta \omega_{3 \mathrm{~dB}}}{\Delta \omega}}{1+\frac{\Delta \omega_{3 \mathrm{~dB}}^{2}}{\Delta \omega^{2}}}
$$

and when $\Delta \omega \ll \Delta \omega_{3 \mathrm{~dB}}$, we see that

$$
\begin{aligned}
R_{\mathrm{hpf}}\left(\Delta \omega \ll \Delta \omega_{3 \mathrm{~dB}}\right) & =i \frac{\Delta \omega}{\Delta \omega_{3 \mathrm{~dB}}} \\
\Rightarrow \quad\left|R_{\mathrm{hpf}}\left(\Delta \omega \ll \Delta \omega_{3 \mathrm{~dB}}\right)\right| & =\left|\frac{\Delta \omega}{\Delta \omega_{3 \mathrm{~dB}}}\right|
\end{aligned}
$$

and thus $\left|R_{\mathrm{hpf}}\right|$ has a $\Delta \omega$ dependence. So now, we have to somehow multiply the baseband response of the hpf to the impedance of the $\mathrm{RF}$ cavity which is strongest about $\omega_{R}$ to obtain a constant impedance. This is where the phase shifter comes in. ${ }^{\dagger}$

A phase shifter is a device takes as input a voltage $V_{\text {in }}$ and converts it to a phase shift $\Delta \phi$ in the RF. Suppose the conversion factor between voltage and angle is $K \mathrm{rad} / \mathrm{V}$ and the RF to be shifted is $V_{\mathrm{RF}} \sin \omega_{\mathrm{RF}} t$ and $V_{\mathrm{in}}(\Delta \omega)=V_{0} e^{i \Delta \omega t} R_{\mathrm{hpf}}(\Delta \omega)$, then from Appendix $I$, the output of the phase shifter is

$$
V_{\Delta \phi}(\text { dipole mode })=\frac{V_{\mathrm{RF}}}{2} K V_{0} R_{\mathrm{hpf}}(\Delta \omega) e^{i \omega t}
$$

from which we can just read off ${ }^{\ddagger}$ the dipole mode response of the hpf phase shifter combination as

$$
\left|R_{\mathrm{hpf}+\Delta \phi}(\omega)\right|=\left|\frac{V_{\mathrm{RF}}}{2} K R_{\mathrm{hpf}}(\Delta \omega)\right| \sim\left|\frac{\Delta \omega}{\Delta \omega_{3 \mathrm{~dB}}}\right|
$$

Therefore, $R_{\mathrm{hpf}+\Delta \phi}(\omega) Z_{\mathrm{RF}}(\omega)$ will have a constant impedance in the region around $\omega_{R} \approx$ $\omega_{\mathrm{RF}}$ and $\left|\omega_{\mathrm{RF}}-\omega\right| \ll \omega_{3 \mathrm{~dB}}$ as required. In the design, we chose $f_{3 \mathrm{~dB}}=2 \mathrm{MHz}$

$\dagger$ We can also just mix up using $\sin \omega_{\mathrm{RF}} t$. However, this is not the setup which we use.

$\ddagger$ There is a subtlety here, since the phase shifter is a non linear device which means technically there is no frequency response. However, by introducing $\Delta \omega$ which is equivalent to a mixed down signal, we can talk of a response. See Appendix II for more discussion of frequency responses for non linear devices. 


\section{Notch Filter}

The notch filter used in the electronics serves a two fold purpose. First, it suppresses the revolution harmonics. Second, it differentiates the synchrotron sidebands around the revolution harmonics which tells the damper which direction to kick. In our setup, the notch filter is created with two digital delay lines. Its response is given by

$$
R_{\text {notch }}(\omega)=1-e^{-i \omega N T}
$$

where $T$ is the revolution period and $N$ is the number of revolution periods in the delay. We will see later in this section that the choice of $N$ is a compromise between the synchrotron frequency of the Tevatron at $150 \mathrm{GeV}$ and $980 \mathrm{GeV}$.

The notch filter clearly suppresses the revolution harmonics at $\omega_{0}=2 \pi f_{0}$ since $R_{\text {notch }}=$ 0 whenever

$$
\begin{gathered}
\omega=2 M \pi / N T \quad M \in \mathbb{Z} \\
f=\frac{M}{N} f_{0}
\end{gathered}
$$

i.e. a notch appears at the revolution harmonic $f_{0}$ whenever $M$ is a multiple of $N$. Another observation is that the number of notches between 0 and $f_{0}$ is $N$.

To show that the slope of the notch around the synchrotron frequency is a differentiator, let us choose an $M=1$ notch at $2 \pi / N T \equiv \omega_{z}$ (Obviously, any $2 M \pi / N T$ will work). The synchrotron frequency near this notch is $\Omega_{s}=\omega_{z}+\omega_{s}$, which means that the response of the notch filter at $\Omega_{s}$ is

$$
\left.\begin{array}{rl}
R_{\mathrm{notch}}\left(\Omega_{s}\right) & =1-e^{-i \Omega_{s} N T}=1-e^{-i \omega_{s} N T} \\
& =2 \sin \frac{\omega_{s} N T}{2} e^{i \varphi}
\end{array}\right\}
$$

where $\tan \varphi=\sin \omega_{s} N T /\left(1-\cos \omega_{s} N T\right)$. Now when $\omega_{z} \neq 0$, we have $\omega_{s} N T=2 \pi \omega_{s} / \omega_{z} \approx 0$ if $\omega_{s} \ll \omega_{z}$, so that $\varphi=\pi / 2$, then

$$
R_{\mathrm{notch}}\left(\Omega_{s}\right) \approx i \omega_{s} N T+\ldots=i\left(\Omega_{s}-\omega_{z}\right) N T+\ldots
$$


which to first order in $\omega_{s} N T$ has differentiated in time the synchrotron sidebands at $\Omega_{s}$.

As mentioned earlier, the choice of $N$ are compromises between the Tevatron's injection energy at $150 \mathrm{GeV}$ and its top energy at $980 \mathrm{GeV}$ and the phase and amplitude responses at these two energies. We chose $N T=1 / 6 f_{s}$ where $f_{s} \approx 88 \mathrm{~Hz}$ is the synchrotron frequency at $150 \mathrm{GeV}$. Therefore, $N=90$ when $T=21 \mu \mathrm{s}$. (Note: we have actually set $N=91$ in the real setup).

\section{Near Uniform Triggers}

In order for the digital delays to work they have to be triggered. The triggers which we use are nearly uniform in time. The reason for the non-uniformity in triggers comes from the spacing of the bunches in the Tevatron. At present, there are three trains of 12 bunches. In each train, the bunches are spaced 21 buckets apart. The spacing between the trains are the abort gaps and they take up 140 buckets each. As a check, we can add these numbers up $3 \times(21 \times(12-1)+140)=1113$ which is exactly the harmonic number of the Tevatron. Notice that 21 does not divide the number of buckets in the abort gap. This observation threw us off initially when we had triggers which were uniformly spaced 21 buckets apart. We fixed this by having triggers at the following bucket locations:

$$
\begin{array}{r|c}
\text { in train } & \text { in abort gap } \\
\mathbf{1}, \mathbf{2 2}, \mathbf{4 3}, \ldots, \mathbf{2 1 1}, \mathbf{2 3 2} & \mid 253,274, \ldots, 337,358 \\
\mathbf{3 7 2}, \mathbf{3 9 3}, \mathbf{4 1 4}, \ldots, \mathbf{5 8 2}, \mathbf{6 0 3} & 624,645, \ldots, 708,729 \\
& \\
\mathbf{7 4 3}, \mathbf{7 6 4}, \mathbf{7 8 5}, \ldots, \mathbf{5 8 2}, \mathbf{6 0 3} & 624,645, \ldots, 1079,1100
\end{array}
$$

which has mostly a 21 bucket spacing with the exceptions being between the last bucket of the abort gap and the first bucket of the train when we only have 14 buckets.

The reason for having near uniform triggers rather than having triggers where the bunches are is to allow us to use reasonable cable delays to ensure that the correct bunches 
are kicked. In the worst case scenario for near uniform triggers, the cable length will be 21 buckets $/ 2 \approx 200 \mathrm{~ns}$ for correctly hitting the right bunch. While for triggers where there are bunches only, the worst case scenario will be 140 buckets $/ 2 \approx 1.3 \mu$ s of cable!

\section{One Turn Delay}

The goal of one turn delay is to ensure that when we detect bunch $n$ we kick bunch $n$ some time later. To ensure that we do indeed do this, we have to find out how the synchrotron sidebands will look like if we have an arbitrary delay in the damper system.

We can think of the synchrotron motion as the execution of simple harmonic motion w.r.t. the revolution harmonics and thus the sidebands around them must have certain symmetry properties. For example, about the revolution harmonic $k \omega_{0}$ where $k \in \mathbb{Z}$, if the sidebands are described by $S(\omega)$, then they are related by

$$
S\left(k \omega_{0}-\delta \omega\right)=S^{*}\left(k \omega_{0}+\delta \omega\right) \quad \text { if }(k-1) \omega_{0} / 2<\delta \omega<(k+1) \omega_{0} / 2
$$

The condition on $\delta \omega$ is to ensure that we do not overlap the sidebands about $(k-1) \omega_{0}$ and $(k+1) \omega_{0}$.

If there is delay $\Delta t$ in the system, then clearly $S(\omega) \rightarrow e^{-i \omega \Delta t} S(\omega) \equiv S(\Delta t, \omega)$. This means that $S(\Delta t, \omega)$ in general, no longer has the symmetry properties of (22) about $k \omega_{0}$ except for special cases. We can take a stab at finding the special cases by considering when $\Delta t$ is exactly an integer multiple $\ell$ of the revolution frequency, i.e. $\Delta t=2 \pi \ell / \omega_{0}$, then

$$
e^{-i \omega \Delta t}=e^{-i\left(k \omega_{0}+\delta \omega\right) 2 \pi \ell / \omega_{0}}=e^{-i 2 \pi \ell \delta \omega / \omega_{0}} \approx 1 \text { when } \delta \omega / \omega_{0} \ll 1 \text {. }
$$

Clearly, in the Tevatron with the synchrotron frequency between $34 \mathrm{~Hz}$ and $88 \mathrm{~Hz}$, and the revolution frequency at $47 \mathrm{kHz}$, the $\delta \omega / \omega_{0}$ condition holds. Thus when we have exactly one turn delay (or multiple turn delays), the sidebands have the symmetry properties given by $(22)$. 
Phase Shifts

There are three devices in the damper circuit which introduce major phase shifts. They are:

(i) RF cavity, around the resonance $\delta \omega \ll \omega_{R}$.

(ii) High pass filter, at base band $\omega \ll \omega_{\mathrm{RF}}$.

(iii) Notch filter, at every revolution harmonic $\omega_{0}$.

Shown in Figure 4 are the magnitudes and phase shift response of each of the devices. For each of the devices we can make the following approximations:

(a) RF cavity: shifts the phase $\pi / 2$ below resonance and $-\pi / 2$ above resonance.

(b) High pass filter: the phase shift is $+\pi / 2$ phase shift when $0<\omega \ll \omega_{3 \mathrm{~dB}}$.

(c) Notch filter: near the notch, the phase shift is $-\pi / 2$ below the notch and $+\pi / 2$ above the notch.

As an example, let us use the response of the synchrotron sidebands at $\omega_{\mathrm{RF}}+\omega_{0} \pm \omega_{s}$ and pass them through the RF cavity. Now the RF cavity rotates the imaginary part according to $(a)$ and thus the real part of the result is anti-symmetric about $\omega_{R}$. When we mix this down, we should measure sidebands about $\omega_{0}$ which have anti-symmetric real parts. See Figure 5(b). Things are actually a bit complicated because mixing is a nonlinear process. In order to have zero phase shift from mixing down, we have to assume that the mixing process occurs in pairs, i.e. the synchrotron sidebands are measured from a mixed down signal which is excited with a mixed up signal. The phase of the signal into the mix down and the excitation which is mixed up is zero.

Next, when the mixed down signal goes through the high pass filter, both sidebands are rotated by $+\pi / 2$, and thus the imaginary part must now be antisymmetric. See Figure $5(\mathrm{c})$. 

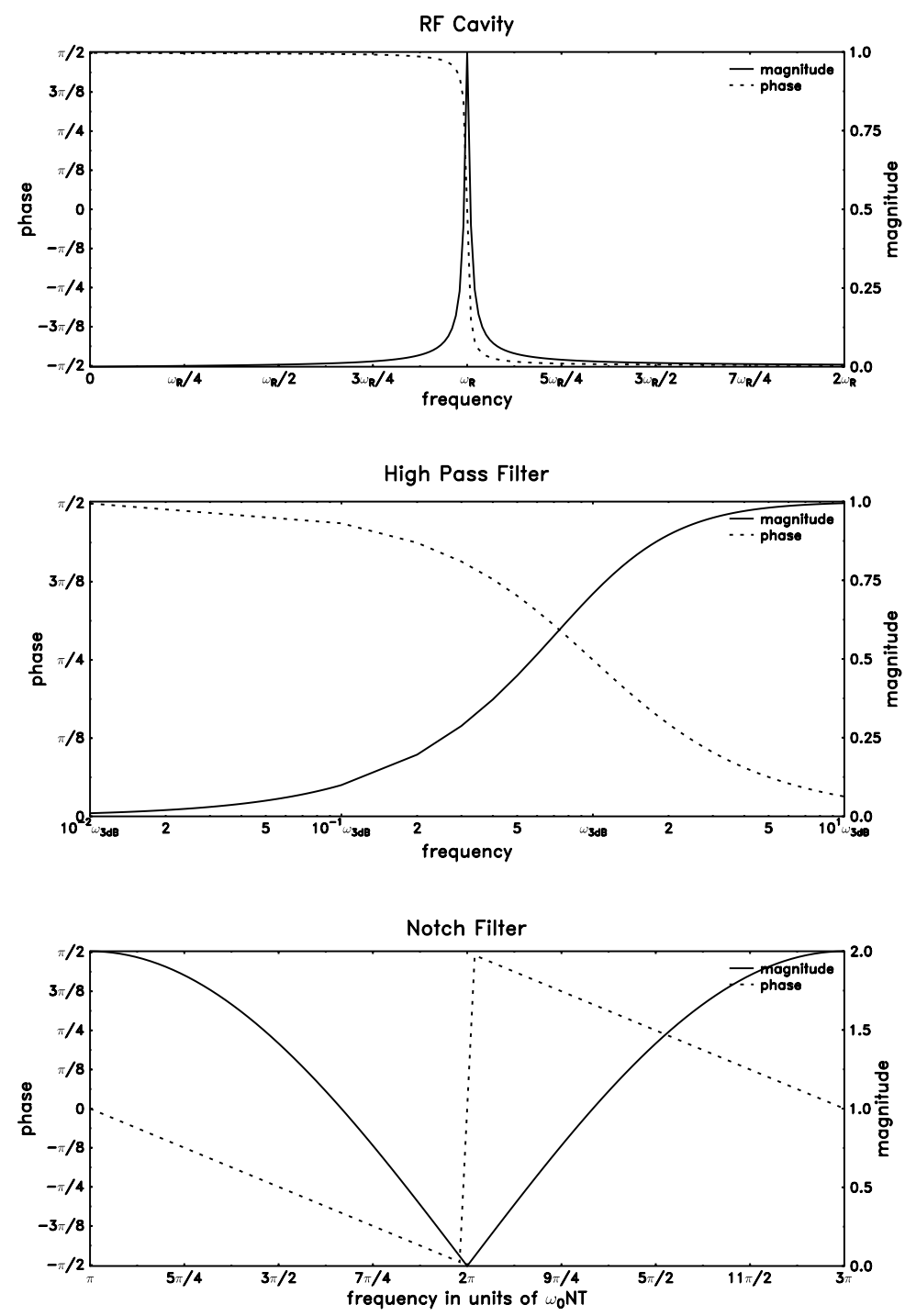

Figure 4 The magnitude and phase responses of three of the devices in the damper circuit.

Finally, when we take this signal and pass it through a notch filter, they become perfectly symmetric! And if we have the sign of the gain right, they will be symmetric and negative which is exactly what is required for damping. See (10) and Figure 5(d). 

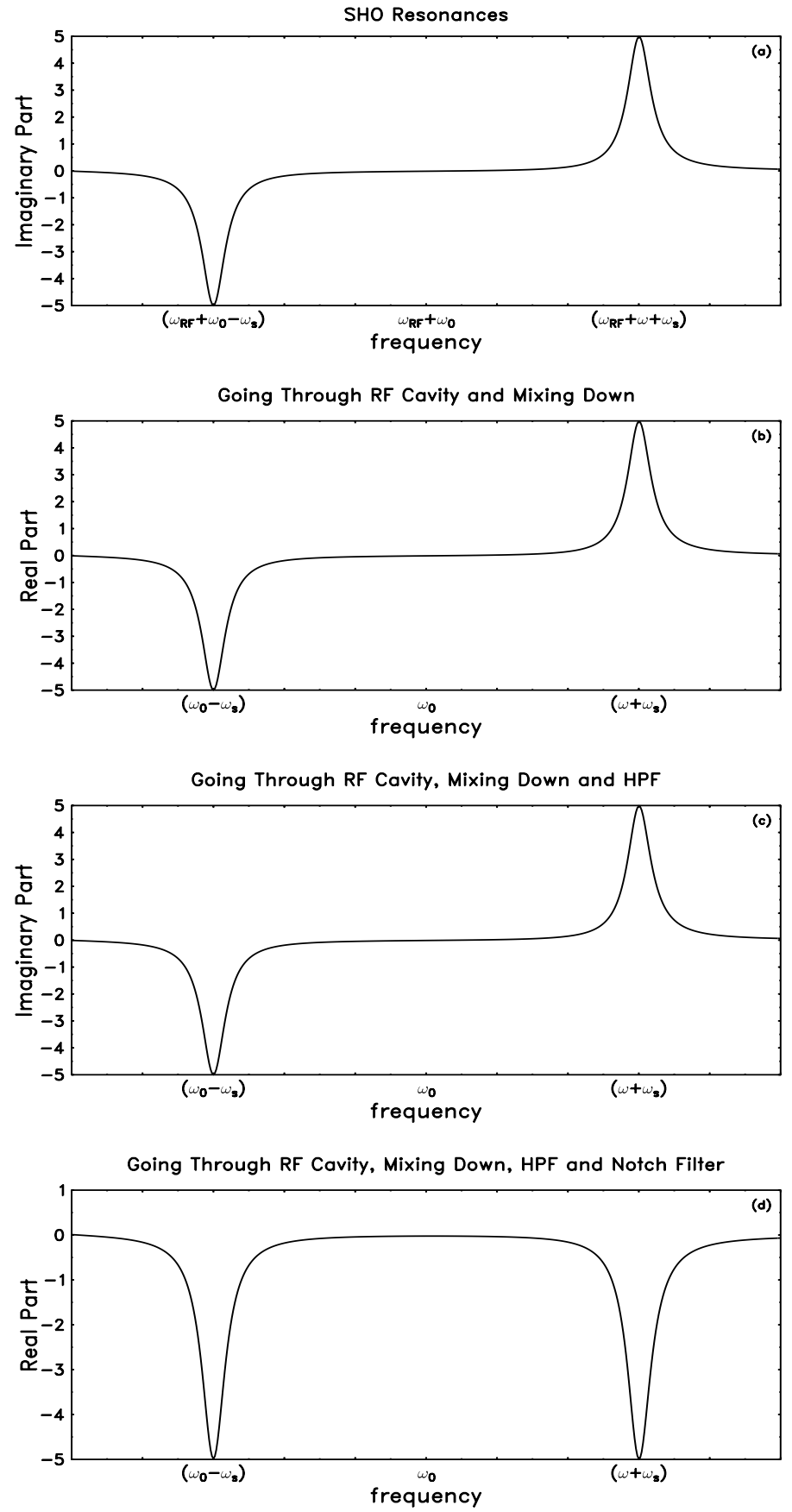

Figure 5 These figures show the upper and lower sidebands of a sho going through each device in the damper. 


\section{Problems with Mode 0}

Although the bunches are not evenly populated in the Tevatron and thus the labels used for coupled bunch modes are not good quantum numbers, we will continue to use them. ${ }^{\dagger}$ The coupled modes are labelled by $\mu$ so that the associated synchrotron frequencies appear at $\left(\mu f_{0} \pm f_{s}\right)$. Since the vectors associated with $\mu$ are not orthogonal, excitation (and hence damping) of mode $\mu$ will result in other modes being excited (or damped). For the dampers, there are only 26 modes which need to be considered because the sampling frequency is $2.5 \mathrm{MHz}$, which means that the highest mode frequency is $1.25 \mathrm{MHz}$ and the highest order mode is $1.25 \mathrm{MHz} / f_{0}=26$.

In fact, the bunch by bunch dampers do not work on mode 0 . If we go through the rotations in phase from each element as we did under Phase Shifts, we will find that the real part of the response is anti-symmetric about $\omega=0$. The source of the problem is that the high pass filter has a phase shift of $-\pi / 2$ when $-\omega_{3 \mathrm{~dB}} \ll \omega<0$. However all is not lost, the gain near $\omega_{0}$ is small because of the hpf and the notch filter and thus mode 0 will not be damped directly. But this mode can still be indirectly damped by using the non-orthogonality of the mode vectors.

$\dagger$ Technically, for unevenly spaced bunches in the Tevatron, we need at least two quantum numbers. An example of a bad set of quantum numbers which can be used are the train number and the bunch number in that train. 


\section{RESULTS}

Finally, we get to look at the results of our measurements. The first set of measurements are performed with delay B disconnected. See Figure 6. This is to get the delay to be exactly 1 turn, i.e. $\Delta t=2 \pi / \omega_{0}$. When this is done, we can get the response to look like Figure 5(c). Three of the possible 26 modes are shown in Figure 7. Before the correct amount of delay is set in Delay A, the as found imaginary part of the response is the top graph in Figure 7. When the delay is made exactly right, we get nice anti-symmetric imaginary responses for all the modes. Three of the modes are shown as examples in the bottom graph of Figure 7 .

With the delay set in Delay A, we can now make the notches by reconnecting back Delay B and by setting the delay in this card by $N(=91)$ revolution periods w.r.t. Delay A. (The value of $N$ was calculated in the subsection Notch Filter). The notches are not quite perfect on each revolution harmonic because the electronics in each card are not exactly the same and so there is some small error in delay $\delta t$. To fix this, we need to add a length of cable between Delay A and Delay B. The length of this cable is determined using the method outlined in Appendix III. This method works really well and we find that the notches will land exactly on the revolution harmonics. However, the two cards do not have exactly the same gain and serendipidously, a shorter cable (and thus a smaller attenuation) actually gives a better notch, albeit not exactly on the revolution harmonic. The results are shown in Figure 8.

Finally, we add in the notch filter into the circuit and voilà, like it was previously discussed and shown in Figure 5(d) with the gain set to -1, the real part of the response is negative and symmetric. These results are shown in Figure 9. The top graph of Figure 9 shows the response at $150 \mathrm{GeV}$ and the bottom graph is at $980 \mathrm{GeV}$.

To test whether the dampers indeed work, we excite the beam at $980 \mathrm{GeV}$ by switching 


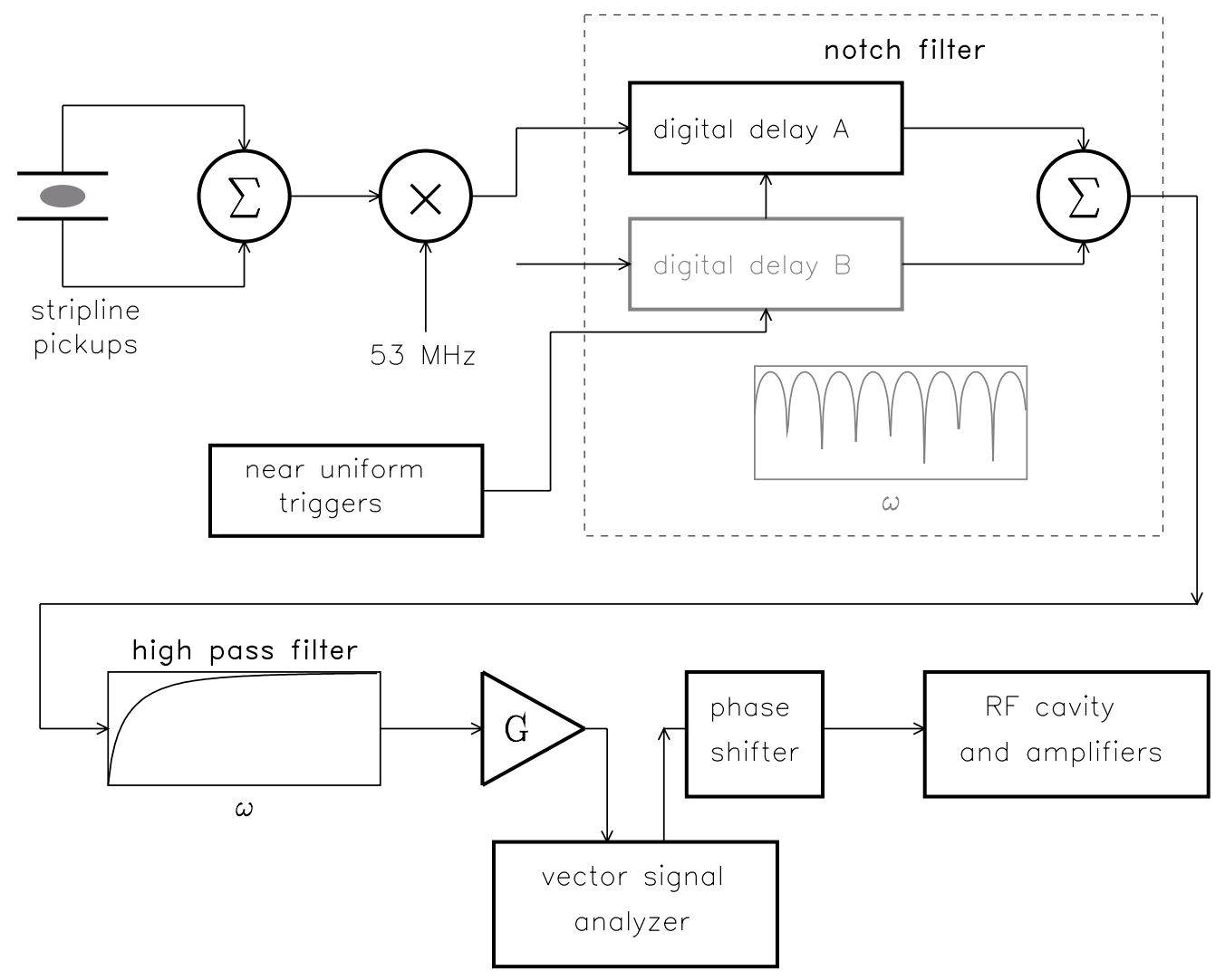

Figure 6 To get the 1 turn delay correct, we disconnected digital delay $\mathrm{B}$ and measured the response. The response of three of the modes are shown in Figure 7.

the sign of the gain. This is a good sign because we can actually excite the beam which means that there is sufficient gain in the loop. When we switch the sign of the gain back to damping, we find that the excitation can be damped. The results of these actions are shown in Figure 10. Although the dampers do perform their job, we find that damping takes 2 to 3 minutes in these examples. 

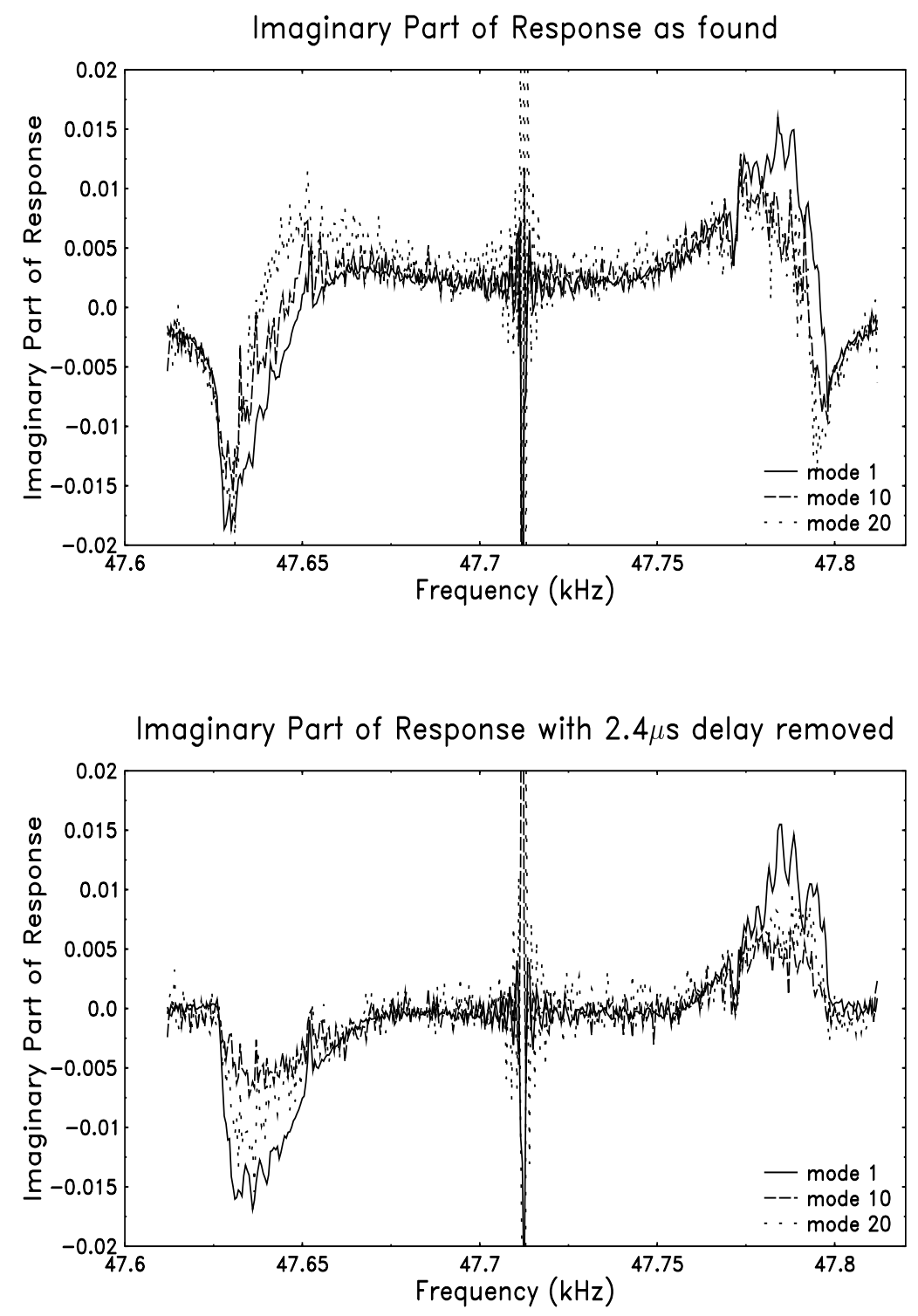

Figure 7 These graphs show the imaginary part of the response of modes 1, 10 and 20 before and after the delay was corrected. We have superimposed all the three graphs on top of each other by shifting the frequency of mode 10 by $-10 f_{0}$ and mode 20 by $-20 f_{0}$. 

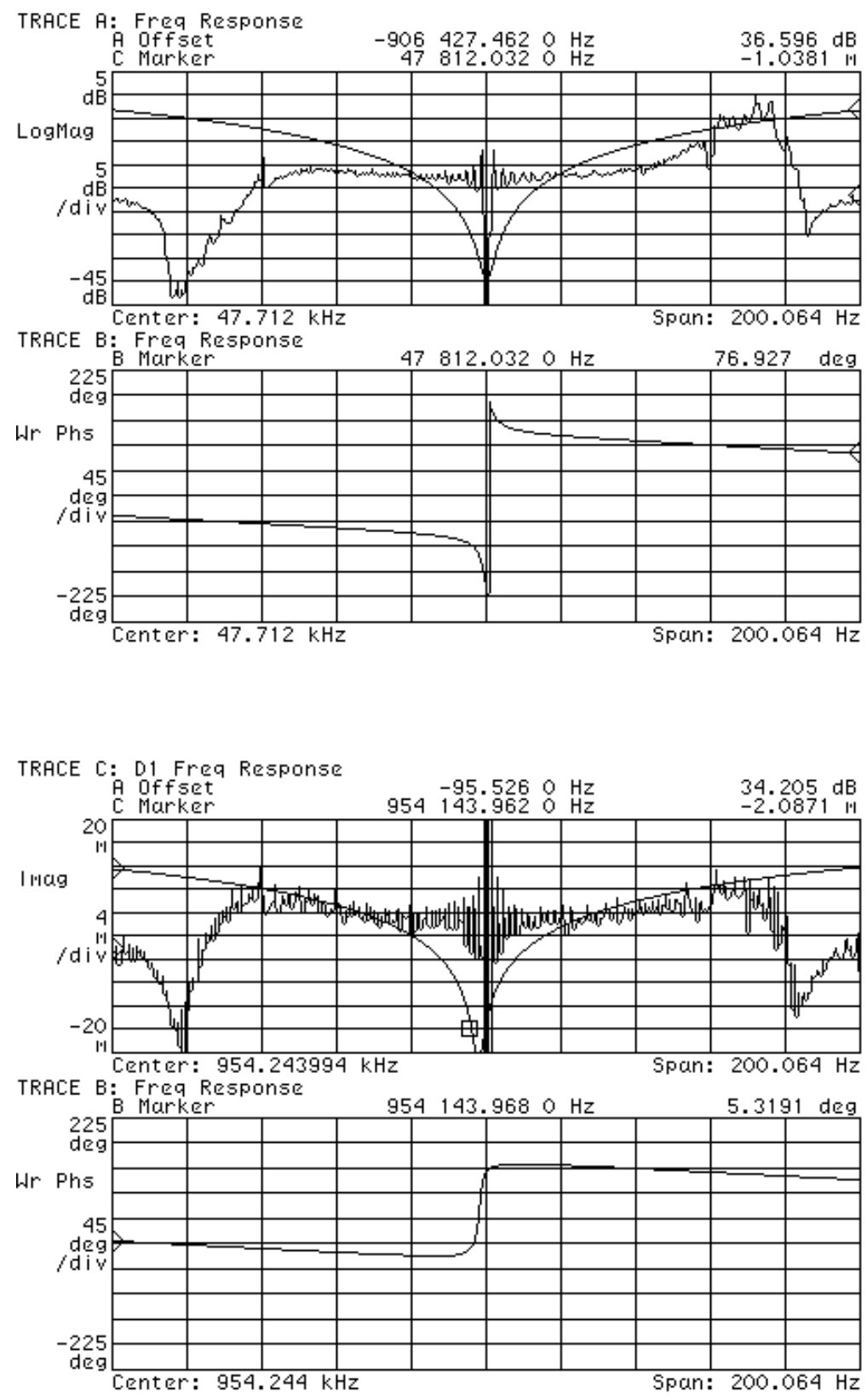

Figure 8 With both delays in the loop, we get notches near the revolution harmonics. The uncorrected imaginary response with one digital delay is superimposed for reference. See text for more details. 

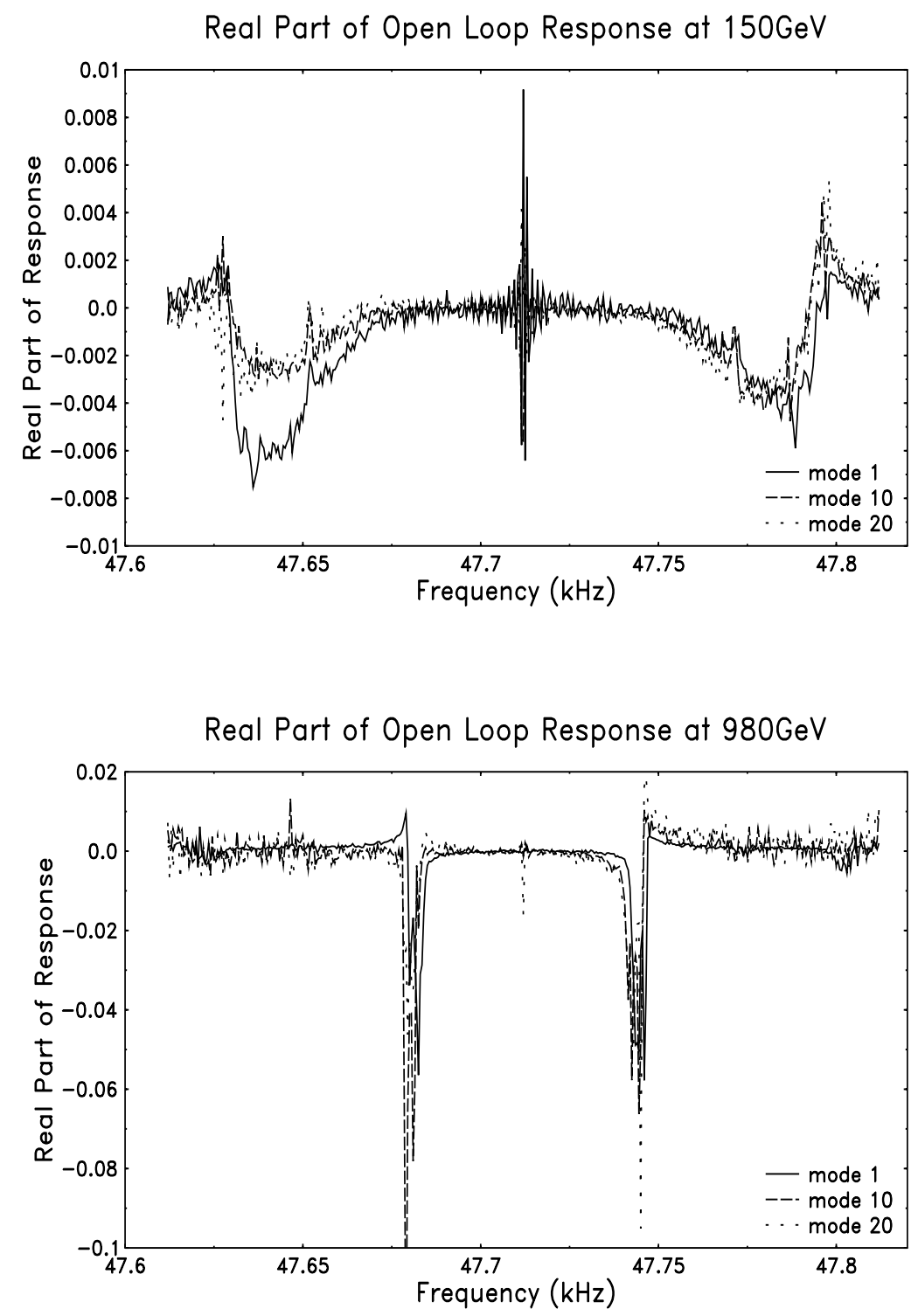

Figure 9 These graphs show the real part of the open loop response of modes 1,10 and 20 at $150 \mathrm{GeV}$ and $980 \mathrm{GeV}$. We have superimposed all the three graphs on top of each other by shifting the frequency of mode 10 by $-10 f_{0}$ and mode 20 by $-20 f_{0}$. 

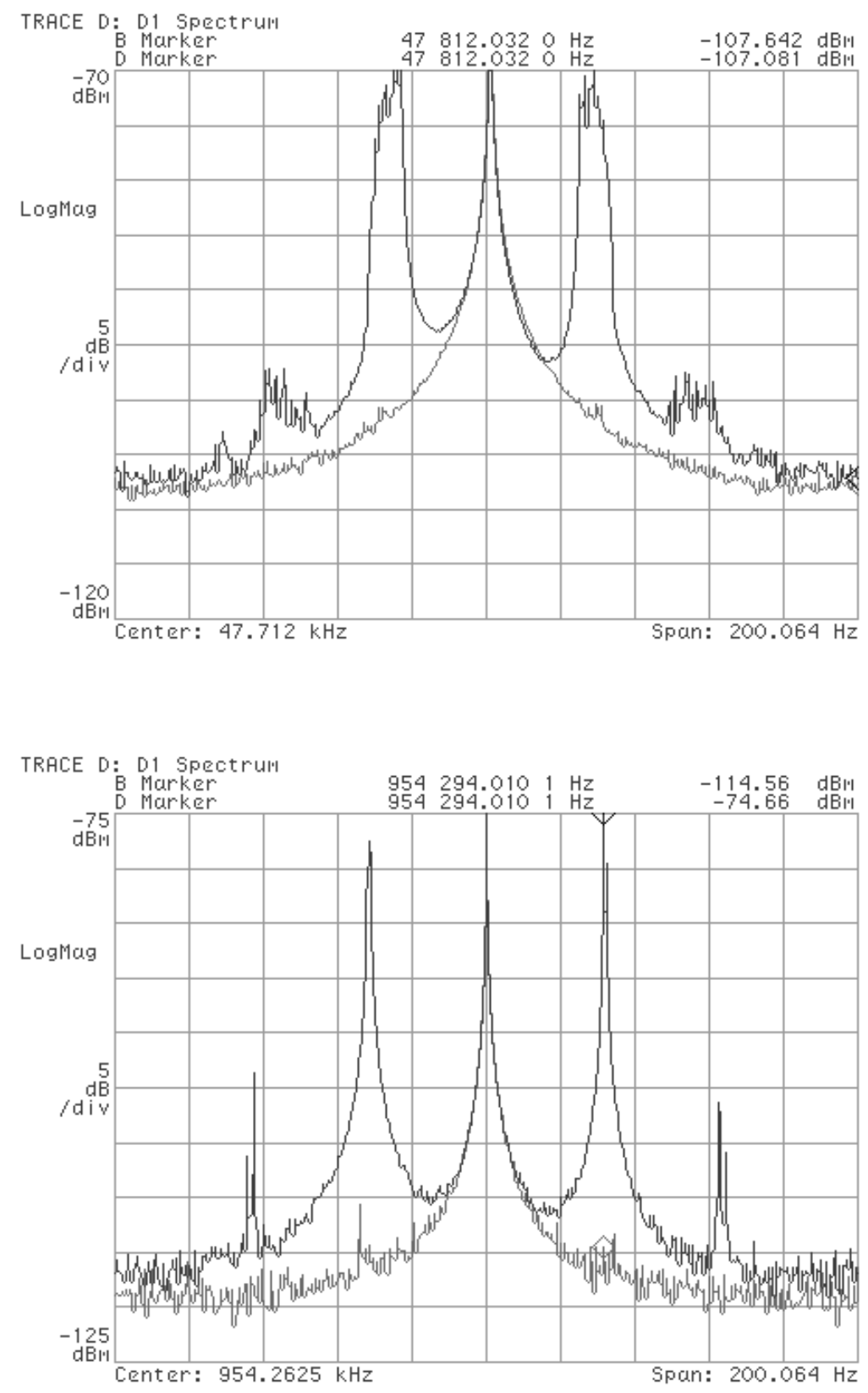

Figure 10 When we closed the loop at $980 \mathrm{GeV}$, we excited the beam by anti-damping it. Then we turned on damping and clearly the synchrotron lines of mode 1 and 20 were damped. 


\section{CONCLUSION}

After installing the dampers, the problem of sudden beam size growth during a store discussed in Introduction, is no longer observed. To prove to ourselves that the dampers definitely stopped the problem, we deliberately turned the dampers off for one store. In this store the beam blew up longitudinally as before. This conclusively showed us that the longitudinal dampers solved the problem. However, the underlying cause of the blowup is still not understood. There are speculations that higher order parasitic modes in the RF cavity, phase noise from microphonics etc. are the source of these blowups. For intellectual satisfaction, a hunt for the source will be the next thing to do. However, operationally, the dampers are a success. 


\section{APPENDIX I}

In this appendix, we will describe the effect of a phase shifter. The phase shifter, like a mixer, is a non linear device. It takes as input a voltage $V_{\text {in }}$ and converts it to a phase shift $\Delta \phi$ in the RF. The goal here is to pick out a term in the action of the phase shifter which is applicable to the calculation of frequency responses. This choice will be made clear in Appendix II.

Suppose the conversion factor between voltage and angle is $K \mathrm{rad} / \mathrm{V}$ and the $\mathrm{RF}$ to be shifted is $V_{\mathrm{RF}} \sin \omega_{\mathrm{RF}} t$ then the output of the phase shifter is

$$
\left.\begin{array}{rl}
V_{\Delta \phi} & =V_{\mathrm{RF}} \sin \left(\omega_{\mathrm{RF}} t+K V_{\mathrm{in}}\right) \\
& =\frac{V_{\mathrm{RF}}}{2 i}\left[e^{i\left(\omega_{\mathrm{RF}} t+K V_{\mathrm{in}}\right)}-e^{-i\left(\omega_{\mathrm{RF}} t+K V_{\mathrm{in}}\right)}\right]
\end{array}\right\}
$$

The input $V_{\text {in }}$ works at base band (in particular, the phase shifter which we use has a bandwidth of $2 \mathrm{MHz}$, and $\left.f_{\mathrm{RF}}=53 \mathrm{MHz}\right)$ and so if we choose $V_{\text {in }}=\tilde{V}_{\text {in }}(\Delta \omega) e^{i \Delta \omega t}$ where $\Delta \omega=\omega-\omega_{\mathrm{RF}}$, then when we substitute this into the first ${ }^{\dagger}$ term of $(24)$

$$
e^{i\left(\omega_{\mathrm{RF}} t+K V_{\mathrm{in}}\right)}=e^{i \omega_{\mathrm{RF}} t} e^{i K \tilde{V}_{\mathrm{in}}(\Delta \omega)(\cos \Delta \omega t+i \sin \Delta \omega t)}
$$

From Gradshteyn, we have

$$
e^{i z \cos \varphi}=\sum_{\ell=-\infty}^{\infty} i^{\ell} J_{\ell}(z) e^{i \ell \varphi}
$$

and

$$
e^{i z \sin \varphi}=J_{0}(z)+\sum_{\ell=1}^{\infty} J_{\ell}(z)\left[e^{i \ell \varphi}+(-1)^{\ell} e^{-i \ell \varphi}\right]
$$

Thus by employing the above properties, we get from (25)

$$
\begin{aligned}
& e^{i\left(\omega_{\mathrm{RF}} t+K V_{\mathrm{in}}\right)}=e^{i \omega_{\mathrm{RF}} t} \sum_{\ell=-\infty}^{\infty} i^{\ell} J_{\ell}\left(K \tilde{V}_{\mathrm{in}}(\Delta \omega)\right) e^{i \ell \Delta \omega t} \times \\
& {\left[J_{0}\left(i K \tilde{V}_{\mathrm{in}}(\Delta \omega)\right)+\sum_{\ell^{\prime}=1}^{\infty} J_{\ell^{\prime}}\left(i K \tilde{V}_{\mathrm{in}}(\Delta \omega)\right)\left[e^{i \ell^{\prime} \Delta \omega t}+(-1)^{\ell^{\prime}} e^{-i \ell^{\prime} \Delta \omega t}\right]\right] }
\end{aligned}
$$

$\dagger \overline{\left.\text { The second term } e^{-i\left(\omega_{\mathrm{RF}} t+K\right.} V_{\mathrm{in}}\right)}$ gives us terms which vanish when averaged in the calculation of frequency responses. See Appendix II. 
We pick out the dipole term $\left(\ell+\ell^{\prime}\right)=1$ and get

$$
\begin{aligned}
\text { dipole term }= & e^{i \omega_{\mathrm{RF}} t}\left[i J_{0}\left(i K \tilde{V}_{\text {in }}\right) J_{1}\left(K \tilde{V}_{\text {in }}\right)+\right. \\
& \left.\sum_{\ell=1}^{\infty} i^{1-\ell} J_{\ell}\left(i K \tilde{V}_{\text {in }}\right)\left[J_{1+\ell}\left(K \tilde{V}_{\text {in }}\right)+J_{1-\ell}\left(K \tilde{V}_{\text {in }}\right)\right]\right] e^{i \Delta \omega t} \\
= & i e^{i \omega_{\mathrm{RF}} t} K \tilde{V}_{\text {in }} e^{i \Delta \omega t}+\mathrm{O}\left(K \tilde{V}_{\text {in }}\right)^{2}
\end{aligned}
$$

Therefore, the dipole term to first order in $K \tilde{V}_{\text {in }}(\Delta \omega)$ of $V_{\Delta \phi}$ of the phase shifter is

$$
\begin{aligned}
V_{\Delta \phi}(\text { dipole }) & =\frac{V_{\mathrm{RF}}}{2} e^{i \omega_{\mathrm{RF}} t} K \tilde{V}_{\mathrm{in}}(\Delta \omega) e^{i \Delta \omega t} \\
& =\frac{V_{\mathrm{RF}}}{2} K \tilde{V}_{\mathrm{in}}(\Delta \omega) e^{i \omega t}
\end{aligned}
$$

which is the linear term in $K \tilde{V}_{\text {in }}(\Delta \omega)$ which we are seeking. 


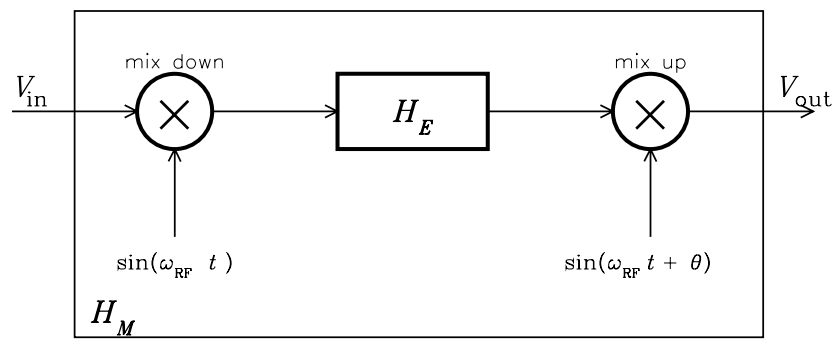

Figure 11 When we have two mixers, one mixing down, the other mixing up, then $V_{\text {out }}(\omega)=H_{M}(\omega) V_{\text {in }}(\omega)$.

\section{APPENDIX II}

The frequency response of a system which contains non-linear devices is in general undefined. However, if they come in appropriate pairs which undo each others non-linearity, then we can define a frequency response. For example, see Figure 11 when we have two mixers, one mixing down and one mixing up. Clearly the input and the output of the system can be described using the frequency response paradigm, i.e. $V_{\text {out }}(\omega)=H_{M}(\Delta \omega) V_{\text {in }}(\omega)$, $\Delta \omega=\omega-\omega_{\mathrm{RF}}$.

In our system, we have a phase shifter which replaces the second mixer shown in Figure 11. We have to show that we can still use the frequency response paradigm. Suppose $V_{\text {in }}=\tilde{V}_{\text {in }} e^{i \omega t}$, then when we mix down with $\sin \omega_{\mathrm{RF}} t=\left(e^{i \omega_{\mathrm{RF}} t}-e^{-i \omega_{\mathrm{RF}} t}\right) / 2 i$, we have at the output of the mixer

$$
V_{\text {mixer }}=-\frac{\tilde{V}_{\text {in }}}{2 i} e^{i\left(\omega-\omega_{\mathrm{RF}}\right) t} \equiv-\frac{\tilde{V}_{\text {in }}}{2 i} e^{i \Delta \omega t}
$$

where we have removed the $e^{i\left(\omega_{\mathrm{RF}}+\omega\right) t}$ term with filters because we are mixing down. Note that we are now at base band, this means that the output after going through $H_{E}$ is

$$
V_{H_{E}}(\Delta \omega)=H_{E}(\Delta \omega) V_{\text {mixer }}=-\frac{\tilde{V}_{\text {in }}}{2 i} H_{E}(\Delta \omega) e^{i \Delta \omega t}
$$


Finally, we can use the results of Appendix I when we go through the phase shifter. We note that terms of $(28)$ when $\left(\ell+\ell^{\prime}\right) \neq 1$ average to zero in the response because they are uncorrelated with the input $V_{\text {in }}$ and so we can ignore them. Therefore, only the dipole term $\left(\ell+\ell^{\prime}\right)=1$ contributes

$$
V_{\text {out }}(\text { dipole })=\frac{i V_{\mathrm{RF}}}{4} K \tilde{V}_{\mathrm{in}} H_{E}(\Delta \omega) e^{i \omega t}
$$

Therefore, the frequency response $H_{M}$ is

$$
H_{M}(\omega)=\frac{i V_{\mathrm{RF}}}{4} K H_{E}(\Delta \omega)
$$

which means that the frequency response paradigm works. 


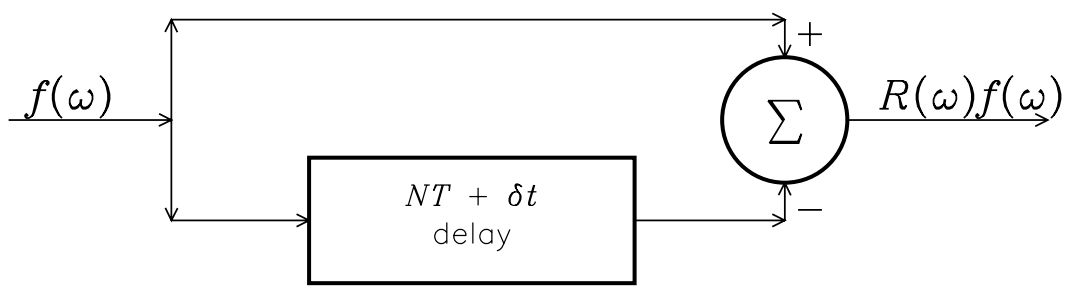

Figure 12 This is a notch filter created using one delay line. The desired delay is $N T$ with an error $\delta t$.

\section{APPENDIX III}

In this appendix, we will show how we can calculate the fine delay $\delta t \mathrm{~s}$ between two digital delay cards when the zeroes of the notch filter are displaced from the desired positions. We can simplify the two digital delay card system into one digital delay card system shown in Figure 12. The system which we will consider has a desired delay $N$ times the sampling period $T$, however, we have also included an undesirable error $\delta t$ which needs to be removed.

We have to write down the frequency response of the notch filter first. This is easily derived and is

$$
R(\omega)=1-e^{-i \omega(N T+\delta t)}
$$

If we define $\omega_{0}=2 \pi f_{0}$ to be the sampling frequency and $\delta \phi=\omega_{0} \delta t$, then

$$
R(\omega)=1-e^{-i(2 N \pi+\delta \phi) \frac{\omega}{\omega_{0}}}
$$

Clearly the zeroes of $R(\omega)$ are when

$$
\left.\begin{array}{c}
\frac{\omega}{\omega_{0}}(2 N \pi+\delta \phi)=2 M \pi \quad \text { where } M \in \mathbb{Z} \\
\Rightarrow \quad \omega \equiv \omega_{z}=\frac{2 M \pi \omega_{0}}{2 N \pi+\delta \phi}
\end{array}\right\}
$$


Therefore, when $\delta \phi=0$, i.e. when there are no errors, we must have the zeroes at

$$
\omega_{z}=\frac{M}{N} \omega_{0}
$$

However, if $\delta t \neq 0$, the notches will be displaced. In particular, if we measure the notch near $M f_{0}$, and find that it is displaced by $\delta f$ w.r.t. $M f_{0}$, then

$$
\left.\begin{array}{r}
\omega_{z}-\frac{M}{N} \omega_{0}=2 \pi \delta f \\
M \omega_{0} \delta \phi \\
-\frac{M(2 N \pi+\delta \phi)}{N(2 N \delta f}
\end{array}\right\}
$$

where we have used (37). Therefore, by solving for $\delta \phi$, we can obtain the error in delay $\delta t=\delta \phi / \omega_{0}$ 


\section{APPENDIX IV}

The impedance of a RF cavity can be modelled using a parallel $R L C$ circuit shown in Figure 13. $R$ is the shunt impedance, $L$ the inductance and $C$ the capacitance of the RF cavity.
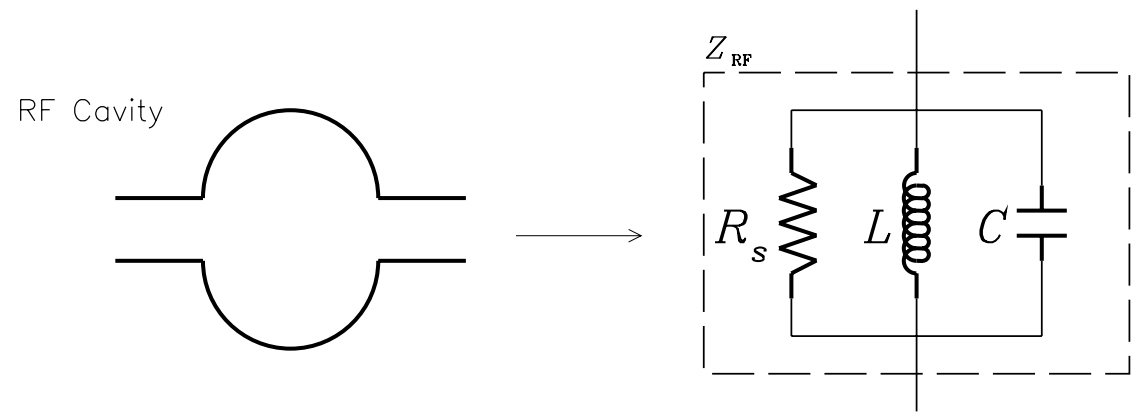

Figure 13 The impedance of an RF cavity is modelled as a parallel $R L C$ circuit.

The impedance $Z_{\mathrm{RF}}$ of the RF cavity is

$$
\frac{1}{Z_{\mathrm{RF}}(\omega)}=\frac{1}{R_{s}}+\frac{1}{i \omega L}+i \omega C
$$

So if $\omega_{R}=1 / \sqrt{L C}$ is its resonant frequency and $Q=R_{s} \sqrt{C / L}$ is its quality factor, then

$$
Z_{\mathrm{RF}}(\omega)=\frac{R_{s}}{1-i Q\left(\frac{\omega_{R}}{\omega}-\frac{\omega}{\omega_{R}}\right)}
$$

Something interesting to note is that (41) differs from Chao (equation 2.81 page 72 ) by a sign in the denominator because of the choice of Fourier transform. If we had used the usual physicist's Fourier definition

$$
\tilde{F}(\omega)=\int_{-\infty}^{\infty} d t e^{i \omega t} f(t)
$$

then the impedance of an inductor which comes from $V=L d I / d t$ is $Z_{L}=-i \omega L$ because $\mathcal{F} \mathcal{T}[d / d t]=-i \omega$. And similarly the impedance of a capacitor $Z_{C}=i / \omega C$. 


\section{High Pass Filter}

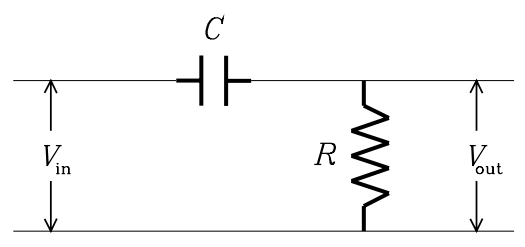

Figure 14 This is a simple high pass filter circuit made of a resistor $R$ and capacitor $C$.

The response $R_{\text {hpf }}$ of a high pass filter (hpf) is modelled with an $R C$ circuit shown in Figure 14 .

$$
V_{\text {out }}=\frac{R}{R+\frac{1}{i \omega C}} V_{\text {in }}
$$

If we define $\omega_{3 \mathrm{~dB}}=1 / R C$, then

$$
R_{\mathrm{hpf}}(\omega)=\frac{V_{\text {out }}}{V_{\mathrm{in}}}=\frac{1+i \frac{\omega_{3 \mathrm{~dB}}}{\omega}}{1+\frac{\omega_{3 \mathrm{~dB}}^{2}}{\omega^{2}}}
$$

\section{REFERENCES}

[1] Introduction to RF Systems, D. McGinnis, 1995 U.S. Particle Accelerator School, Duke University, 1995.

[2] Table of Integrals, Series and Products, I.S. Gradshteyn and I.M. Ryzhik, Academic Press, 2000.

[3] Physics of Collective Beam Instabilities in High Energy Accelerators, A.W. Chao, Wiley Series in Beam Physics, 1993. 